\title{
Progress Towards Better Trauma Care in Under-Privileged Countries
}

\author{
Martin Schuler, ${ }^{1}$ and Suthorn Bavonratanavech ${ }^{2, *}$ \\ ${ }^{1}$ Director Clinical Investigation and Documentation Institute (AOCID), Member of Executive Management at AO Foundation, AO Foundation, University of Rochester - \\ William E. Simon Graduate School of Business Administration, Zurich Area, Switzerland \\ ${ }^{2}$ Past President of AO Foundation, Chief of Orthopedic and Trauma Network, BDMS, Senior Director of Bangkok Orthopedic Center \\ "Corresponding author: Suthorn Bavonratanavech, MD., Bangkok Medical Center, 2 Soi Soonvijai 7, New Petchaburi Rd. Bangkok, 10310, Thailand. Tel: +66-23103192, Fax: \\ +66-27551465, E-mail: suthorn@gmail.com; suthorn.bavonratanavech@ aofoundation.org
}

Received 2016 August 27; Accepted 2016 September 20.

In 1996, the world health organization (WHO) published a landmark booklet, entitled "global burden of disease" (GBD). In this booklet, it was reported that an "epidemiologic transition" is underway. In addition, it was stated that in developing regions, the incidence of death caused by injuries will approach that of communicable and nutritional diseases by 2020 (1).

In 1990, about 5 million people died of injuries of different types. If no effective measures are taken, road traffic accidents (RTA) will be ranked the third cause of disease burden worldwide (1). However, these figures fail to describe the problems caused by injuries in developing countries. From 1980 to 1995, the incidence of RTA deaths decreased by nearly $20 \%$ in high-income countries, while the incidence rate almost doubled in Asian regions (2). Moreover, death due to injuries disproportionally affects men, who are traditional breadwinners and family protectors in developing countries. RTA is the leading cause of death among men aged 15 - 44 years, and RTA deaths pose a significant risk to the economies of developing countries.

GBD brought injuries to the foreground as a serious public health concern. Following its publication, various efforts have been made in both developed and developing countries. One noticeable aspect of improvement is finding better and more efficient ways of handling and caring for trauma victims. Another important development is the understanding that injuries are a preventable public health problem (3). By categorizing injuries as an epidemic, we need to deploy a 2-pronged approach, involving trauma care and trauma prevention, both requiring a good infrastructure which is lacking in developing countries.

1. How Does One Provide Trauma Care in Developing Countries?

With the shortage of trained personnel and lack of physical infrastructure, most trauma care measures, developed in high-income countries, are difficult to apply in low- and middle-income countries. Through acknowledgment of this problem, a flurry of activities has taken place, such as trauma care education in Egypt (4) and trauma management courses in sub-Saharan Africa (5). In the development of these courses, healthcare professionals have partnered with local physicians and authorities. These courses account for infrastructure constraints and local practices to develop usable protocols for trauma care in both pre- and post-hospital phases. Further help is also on the way. The AO Alliance Foundation has recently opened trauma care facilities in both Ghana (6) and Malawi (7). These are just some examples of initiatives, which are either ongoing or underway.

\section{How Does One Prevent Trauma?}

It is important to realize that the major causes of injuries not only vary from one country to another, but also differ among different populations within a given country. For instance, while in developing Asian countries, RTA is the leading cause of death among young men, in SubSaharan Africa, the main cause of injury-related death is war among both men and women (1). Given this heterogeneity, it is important to understand the root causes of injuries so that local and national authorities can begin to design preventative measures. This has been accomplished successfully in the United States through trauma registries. The lessons learned should be shared and adjusted for the environment of less affluent countries (8). A number of projects have been started in this direction. To name a few, a trauma registry system was established in the United Arab Emirates in 2003, and a pilot program has just started this year by the AO Alliance Foundation to establish a district-level trauma registry system in Malawi.

Since the 1990's, more studies have been performed on the global burden of injuries. A study published in 2016 showed that the death toll of injuries has dropped from around 5 million in 1990 to 4.8 million in 2013 (9). In a way, the world has become a safer place for everyone. However, the authors of GBD pointed out that this improvement occurred mainly in high-income countries. Taking a closer look, one realizes that the disability-adjusted life year (DALY) rate has increased in South Asia, as well as West and South sub-Saharan Africa. One has to conclude that further efforts should be made in these under-privileged regions.

On the $20^{\text {th }}$ anniversary of the first publication of GBD, I would like to encourage the readership and perspective 
authors of the Journal of Orthopedic and Spine Trauma to think of the bigger picture and make a difference. We should participate in and encourage our colleagues to attend educational programs, join and establish trauma registries, and communicate with public health authorities. There are still a lot of shortcomings. Hopefully, in the coming 20 years, on the $40^{\text {th }}$ anniversary of the first GBD, we can show that traumas are better handled and managed in under-privileged areas around the world.

\section{References}

1. Murray CJ, Lopez AD. Global burden of disease. 1. Harvard University Press Boston; 1996.

2. Nantulya VM, Reich MR. The neglected epidemic: road traffic in juries in developing countries. BMJ. 2002;324(7346):1139-41. doi: 10.1136/bmj.324.7346.1139. [PubMed: 12003888].

3. Peden MM, McGee K, Krug E, editors. Injury: a leading cause of the global burden of disease, 2000. Geneva: World Health Organization; 2002.
4. El-Shinawi M, McCunn M, Sisley AC, El-Setouhy M, Hirshon JM. Developing sustainable trauma care education in Egypt: sequential trauma education program, steps to success.J Surg Educ. 2015;72(4):e29-32. doi: 10.1016/j.jsurg.2014.12.001. [PubMed: 25600355].

5. Peter NA, Pandit H, Le G, Nduhiu M, Moro E, Lavy C. Delivering a sustainable trauma management training programme tailored for low-resource settings in East, Central and Southern African countries using a cascading course model. Injury. 2016;47(5):1128-34. doi: 10.1016/j.injury.2015.11.042. [PubMed: 26725708].

6. Big step towards better trauma care in Ghana [cited 19 Aug]. Available from: http://ao-alliance.org/2016/07/big-step-towards-bettertrauma-care-ghana/.

7. Doubling surgical capacities in Malawi [cited 19 Aug]. Available from: http://ao-alliance.org/2016/04/doubling-surgical-capacitiesmalawi/.

8. Nwomeh BC, Lowell W, Kable R, Haley K, Ameh EA. History and development of trauma registry: lessons from developed to developing countries. World JEmerg Surg. 2006;1:32. doi: 10.1186/1749-7922-1-32. [PubMed: 17076896].

9. Haagsma JA, Graetz N, Bolliger I, Naghavi M, Higashi H, Mullany EC, et al. The global burden of injury: incidence, mortality, disabilityadjusted life years and time trends from the Global Burden of Disease study 2013. Inj Prev. 2016;22(1):3-18. doi: 10.1136/injuryprev-2015-041616. [PubMed: 26635210]. 